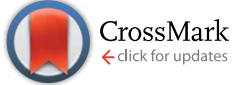

Cite this: Soft Matter, 2014, 10, 8420

Received 16th July 2014

Accepted 1st September 2014

DOI: $10.1039 / c 4 s m 01569 j$

www.rsc.org/softmatter

\title{
Temperature dependent cubic and hexagonal close packing in micellar structures
}

\author{
Nicole Wolff, ${ }^{a}$ Stefan Gerth, ${ }^{a}$ Philipp Gutfreund ${ }^{b}$ and Max Wolff ${ }^{\star c}$ \\ The interfacial structure and phase diagram of a micellar solution formed by the three block copolymer \\ $\left(\mathrm{EO}_{20}-\mathrm{PO}_{70}-\mathrm{EO}_{20}\right)$ also known as $\mathrm{P} 123$ solved in deuterated water close to a solid boundary is \\ investigated with respect to temperature. We find a hysteretic behavior of the $d$-spacing of the micellar \\ crystal and a spontaneous change in the lateral correlation length going hand in hand with a structural \\ reorganization between cubic and hexagonal. The phase transitions may be initiated by a change in the \\ shape of the micelles from spherical to elongated together with a minimization of the polymer water \\ interface.
}

\section{Introduction}

Downsizing technology implies structuring materials on mesoscopic length scales. For a surface this can be achieved by lithographic techniques ${ }^{1}$ or self-assembly., ${ }^{2,3}$ The latter can be used for three dimensional materials, such as photonic crystals, as well. In order to tailor specific properties of these materials and to optimize their performance a detailed understanding of the underlying physics is desirable. Additionally, from a fundamental point of view, micellar self-organization offers a model system to understand phase transitions in weakly interacting systems. ${ }^{4}$

In this context amphiphilic block copolymers are of great interest since they have a strong tendency to phase separation. This is related to the relatively weak interaction potential which is on the order of $k_{\mathrm{B}} T$ at room temperature. ${ }^{5}$ The interaction between molecules or even parts of the molecules with a solvent can be tuned continuously by $e . g$. varying temperature or pressure. Phase separation may result in agglomerates of molecules called micelles of different size and shape. Reaching a critical volume fraction the micelles crystallize in various structures and show complex phase diagrams.

Neutron scattering is a well-established technique for the investigation of soft matter systems. ${ }^{6}$ The remarkable sensitivity of neutrons to light elements and, in particular, the distinct scattering properties of the two hydrogen isotopes ${ }^{1} \mathrm{H}$ and ${ }^{2} \mathrm{H}$ offer the possibility of contrast variation when replacing normal water by heavy water as solvent. Mesoscopic structures can be explored by small angle neutron scattering (SANS). For micellar

${ }^{a}$ Crystallography and Structural Physics, University of Erlangen-Nürnberg, 91058 Erlangen, Germany

${ }^{b}$ Institut Laue-Langevin, B.P. 156, 38042 Grenoble, France

${ }^{c}$ Department of Physics and Astronomy, Uppsala University, 75121 Uppsala, Sweden. E-mail: max.wolff@physics.uu.se systems the structural arrangements ${ }^{7-11}$ as well as the form factor of the micelles ${ }^{12-14}$ have been extracted and phase diagrams with respect to temperature and concentration have been established. ${ }^{15,16}$ By the use of a grazing incident beam geometry SANS experiments can be tuned to become surface sensitive in order to investigate the influence of a confining boundary. ${ }^{17,18}$ We have shown that the symmetry of the unit cell as well as texture and the crystallite size in micellar systems can be investigated by grazing incidence small angle neutron scattering (GISANS). ${ }^{\mathbf{1 9 2 0}}$ For similar copolymer systems we found a distinct hysteresis in the crystalline structure depending on either heating or cooling the sample. ${ }^{21,22}$ In the present work we relate this hysteresis to in-plane correlations investigated via Rocking scans and to the $d$-spacing along the surface normal. Our results are compared to experimental and theoretical phase diagrams presented earlier.

\section{Sample}

The sample under investigation is the tri-block copolymer Pluronic P123 which consists of a central part of 70 propylene oxide units terminated by two end groups of 20 ethylene oxide units $\left(\mathrm{EO}_{20}-\mathrm{PO}_{70}-\mathrm{EO}_{20}\right)$. At low temperatures PPO and PEO show good solubility in water. With increasing temperature the PPO undergoes conformational changes towards lower polarity resulting in a loss of hydration of the polymer chains in water. $^{\mathbf{2 4 , 2 5}}$ On the contrary the PEO blocks maintain their hydrophilicity across a broad range of temperatures. Providing a sufficient polymer concentration and above the critical micellization temperature, these different preferences of the polymer blocks drive the macromolecules to aggregate into micelles with a hydrophobic core (PPO) surrounded by a more hydrophilic shell (PEO). ${ }^{\mathbf{1 6}}$ If the critical volume fraction is reached, these agglomerates may assemble into crystalline structures. ${ }^{15}$ The phase diagram of P123 (see Fig. 1) with respect 


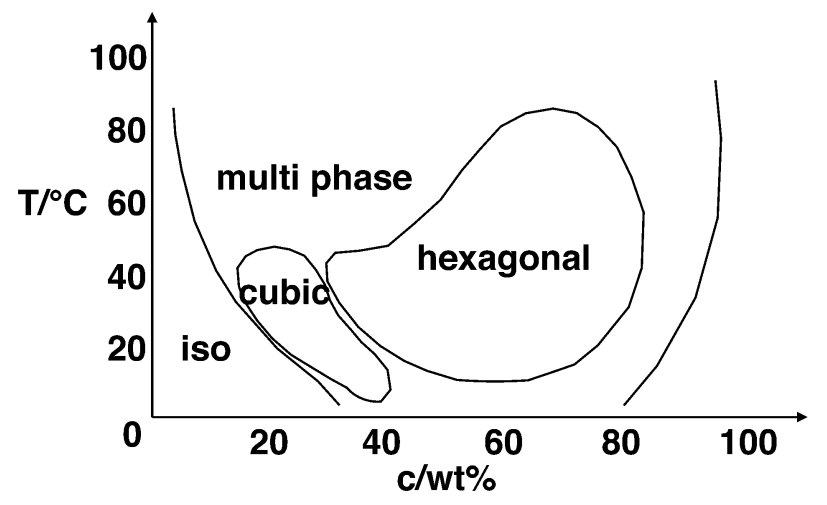

Fig. 1 Experimentally measured phase diagram of P123 solved in water reproduced from ref. 15.

to temperature and concentration was established in ref. 15 and exhibits a cubic and a hexagonal phase for the micellar crystal. We found that the transition between the cubic and the hexagonal phase shows a pronounced thermal hysteresis. ${ }^{22}$

The P123, purchased from Sigma-Aldrich, was used without further purification. The polymer was solved by $28 \%$ in weight in deuterated water for an increased scattering contrast for neutrons (molar ratio equivalent to $30 \% \mathrm{P} 123$ in $\mathrm{H}_{2} \mathrm{O}$ ) at a low temperature of $6{ }^{\circ} \mathrm{C}$ under constant stirring until a homogenous solution was formed. Subsequently the sample was filled into the pre-cooled sample cell in the liquid state $\left(T<14{ }^{\circ} \mathrm{C}\right)$ to prevent shearing of the crystalline phase. To provide a welldefined interface, a silicon wafer was terminated with a hexamethyldisilazane (HMDS) coating adsorbed from the gaseous phase, resulting in a contact angle to water and air of approx. $75^{\circ}$. The neutron data have been recorded while heating the sample from $5{ }^{\circ} \mathrm{C}$ to $66^{\circ} \mathrm{C}$ and subsequent cooling to $5^{\circ} \mathrm{C}$. From rheometry measurements we know that the transition temperature between cubic and hexagonal phase does not depend on the heating and cooling rate.

\section{Experimental details}

All measurements were carried out at the reflectometer ADAM at the Institut Laue-Langevin (Grenoble, France). ${ }^{26}$ Fig. 2 shows the scattering geometry, schematically. The incident neutron beam penetrates a silicon wafer through the narrow side. After

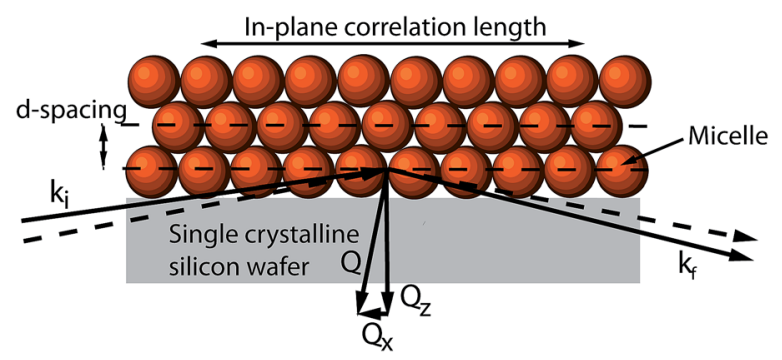

Fig. 2 Schematic illustration of the scattering geometry. The solid and dashed lines symbolize diffuse and specular scattering geometry, respectively. reflection at the solid-liquid interface and scattering at the micellar lattice the intensity is registered by a position sensitive detector (PSD). The dashed arrows in the figure symbolize specular reflectivity (incident beam angle equal to the exit beam angle) with information about the density profile along the surface normal. The geometry to access diffuse scattering, providing information on the in-plane correlations in the micellar structure with different incident and exit beam angles, is symbolized by the solid arrows. The scattering geometry is described in ref. 27 in more detail.

The sample was confined in a liquid cell with the surface treated silicon wafer on one side and an aluminum plate on the other. The total sample thickness was $1 \mathrm{~mm}$, however, due to the scattering geometry the neutron beam penetrates only a few tenth of micrometer from the silicon surface into the liquid. The two plates were sealed by a cadmium spacer and temperature control was realized by circulating coolant from a chiller thru an aluminum cooling attached to the silicon wafer. The design of the sample cell is described in ref. 28.

The incident beam was collimated by slits of $1 \mathrm{~mm}$ at a distance of $2102 \mathrm{~mm}$ along the horizontal direction resulting in a divergence of $0.057^{\circ}$ (FWHM). However, for an incident angle of $0.9^{\circ}$ the resolution is determined by the sample footprint and is $0.04^{\circ}$ or $\Delta Q_{z}=0.02 \mathrm{~nm}^{-1}$ and $\Delta Q_{x}=3 \times 10^{-4} \mathrm{~nm}^{-1}$. Along the vertical axis the full profile from the focusing monochromator was used. The wavelength was $0.441 \mathrm{~nm}$. A position sensitive detector (PSD) collected the specular and diffusely scattered intensity. In this way both the depth profile of the scattering length density and the in-plane correlations are probed via neutron reflectivity $\left(Q_{z}\right)$ and diffuse scattering $\left(Q_{x}\right)$, respectively (see Fig. 2). The out-of-plane structures are resolved on a $\mathrm{nm}$ length scale, whereas diffuse scattering probes a $\mu \mathrm{m}$ length scale in the plane of the interface along the $x$-direction. ${ }^{27}$ By varying the incident beam angle in the given set-up a depth of up to $50 \mu \mathrm{m}$ in the sample from the interface is probed.

The center of the detector was set to a scattering angle of $1.82^{\circ}$ and fixed during all measurements. Since the PSD at ADAM covers $6^{\circ}$ in scattering angle the specular reflected intensity was always monitored. The incident beam angle was varied from $0-1.82^{\circ}$ while continuously heating or cooling the sample. One scan took $10 \mathrm{~min}$ and the heating rate was $0.2{ }^{\circ} \mathrm{C}$ per minute. As a result the sample temperature changes by $2{ }^{\circ} \mathrm{C}$ during one measurement of a Rocking scan.

\section{Results}

Fig. 3 depicts raw data for a typical neutron reflectivity and Rocking scan. The logarithm of the intensity integrated along the $y$-direction is plotted as a color map versus the incident beam angle and the scattering angle. For low incident beam and scattering angles the region of total external reflection is visible. The specular reflectivity can be seen along the diagonal, where the scattering angle equals twice the incident beam angle and is marked by a solid line. The insert on the top left side shows the reflectivity extracted from the data plotted on a logarithmic scale versus $Q_{z}$. The background integrated for an offset incident and exit angle of $0.25^{\circ}$ is represented by the black circles. For 


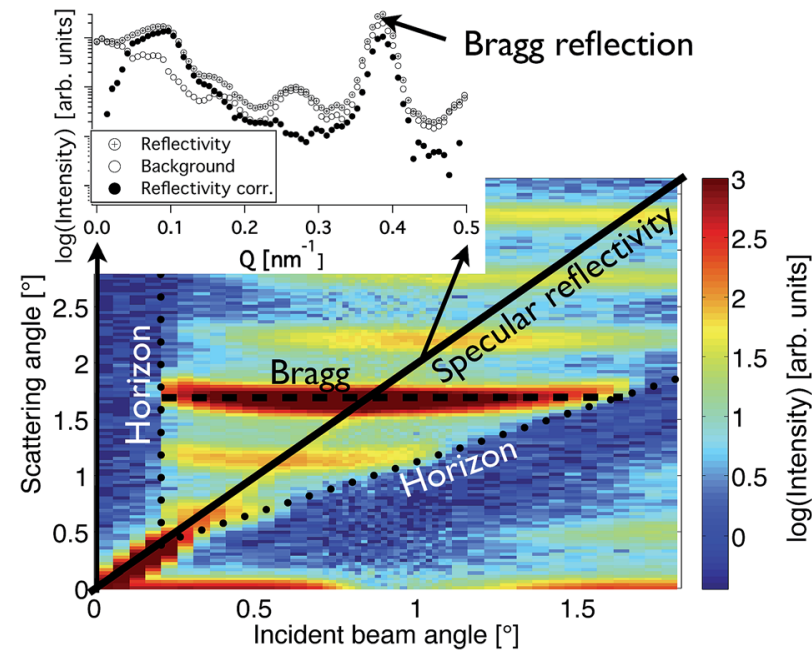

Fig. 3 Typical raw data for a reflectivity measurement plotted as intensity map on a log scale versus the incident and scattering angle. The sample is a $28 \%$ solution of P123 in deuterated water in the crystalline phase in contact with a HMDS-coated silicon surface at a temperature of $17^{\circ} \mathrm{C}$. The specular reflectivity, Bragg reflection and sample horizon are marked by solid, dashed and dotted lines, respectively. The insert in the upper left corner represents the specular reflectivity together with the background.

$Q_{z}<0.3 \mathrm{~nm}^{-1}$ the reflectivity is dominated by small angle scattering. ${ }^{27}$ Bragg reflections visible as shallow lines in the intensity color map have a finite momentum transfer along $Q_{y}$ and become visible because of the relaxed instrumental resolution along the $y$-direction. ${ }^{19}$ The bright horizontal reflection (marked by a dashed line) at a scattering angle of $1.82^{\circ}$ is the first Bragg reflection cutting the specular line. For a detailed discussion of the indexing and relation between real and reciprocal space we refer the reader to one of our earlier publications. ${ }^{19,27}$ In this article we focus on the first order specular peak and will discuss the Rocking scans in detail.

Fig. 4 depicts Rocking curves at the first order Bragg reflection plotted as color map over the incident beam angle and temperature. Below $14{ }^{\circ} \mathrm{C}$ the sample is in the micellar phase with no crystalline ordering resulting in vanishing intensity. For intermediate temperatures the increased intensity is clearly visible indicating the sample being in a crystalline phase. Regions with cubic and hexagonal structural ordering can be separated..$^{15}$ However, the temperature ranges in which these phases exist depend on the thermal history of the sample: ${ }^{22}$ In the heating cycle a dominant cubic ordering can be determined between $14{ }^{\circ} \mathrm{C}$ and $46{ }^{\circ} \mathrm{C}$, and a hexagonal phase between $46^{\circ} \mathrm{C}$ and $61^{\circ} \mathrm{C}$. Coming from high temperatures the micellar crystal first shows a hexagonal ordering starting from $48{ }^{\circ} \mathrm{C}$ which transforms to a cubic structure at $25{ }^{\circ} \mathrm{C}$. All Rocking curves in Fig. 4 consist of a narrow peak (resolution limited, at the position of the specular reflectivity) and broad diffuse scattering related to the correlations in the micellar crystal parallel to the solid-liquid interface. ${ }^{23}$ As outlined above, the temperature is changing during one scan by about $2{ }^{\circ} \mathrm{C}$. In addition as seen in Fig. 4 the intensity along the Rocking scan is strongly

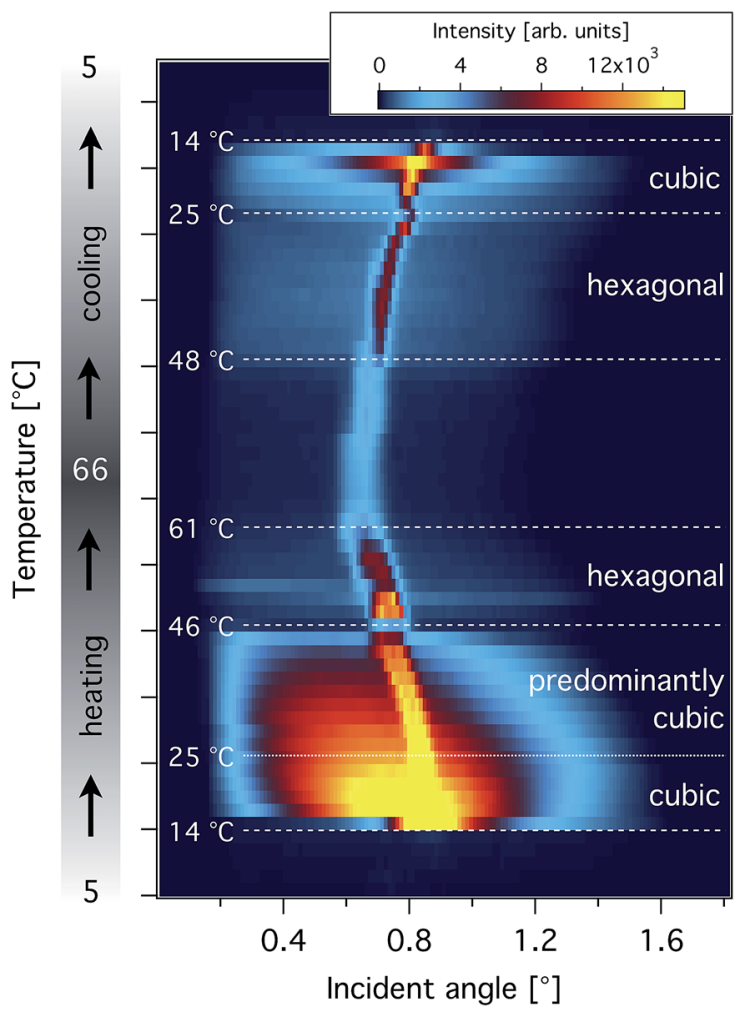

Fig. 4 Intensity map of the Rocking data taken with a $28 \%$ solution of P123 in deuterated water, in contact with HMDS-coated silicon, during a heating and subsequent cooling cycle.

temperature dependent. For example, while heating in the cubic phase the diffuse scattering gets constantly reduced with increasing temperature. This fact can be explained by the asymmetry in the Rocking scans since the temperature during heating was always lower for smaller Rocking angles. $\dagger$

\section{Discussion}

Fig. 5 (upper panel) depicts two Rocking curves taken at $15{ }^{\circ} \mathrm{C}$ and $17{ }^{\circ} \mathrm{C}$, during heating, slightly above the crystallization temperature. The two components mentioned above can be separated for the data taken at $17^{\circ} \mathrm{C}$. The triangular symbols in the lower panel depict the intensity of the narrow (specular) component which was extracted by fitting a Gauss function with sloping background taking the asymmetry of the diffuse signal into account. It turns out that the intensity of this peak remains almost unchanged for heating in the cubic phase. Only at $15^{\circ} \mathrm{C}$ the intensity of the specular component is increased. However, at this temperature the diffuse scattering, as seen in Fig. 5 (upper panel), is narrow, too, with a width comparable to the specular component. Accordingly, the height of the Gaussian line fitted to the data is strongly correlated to the diffusely scattered intensity and the error in the fit, which is usually

$\dagger$ From other measurements we know that additional factors like the footprint, the penetration depth or lateral inhomogeneities can affect the symmetry of a Rocking scan. 

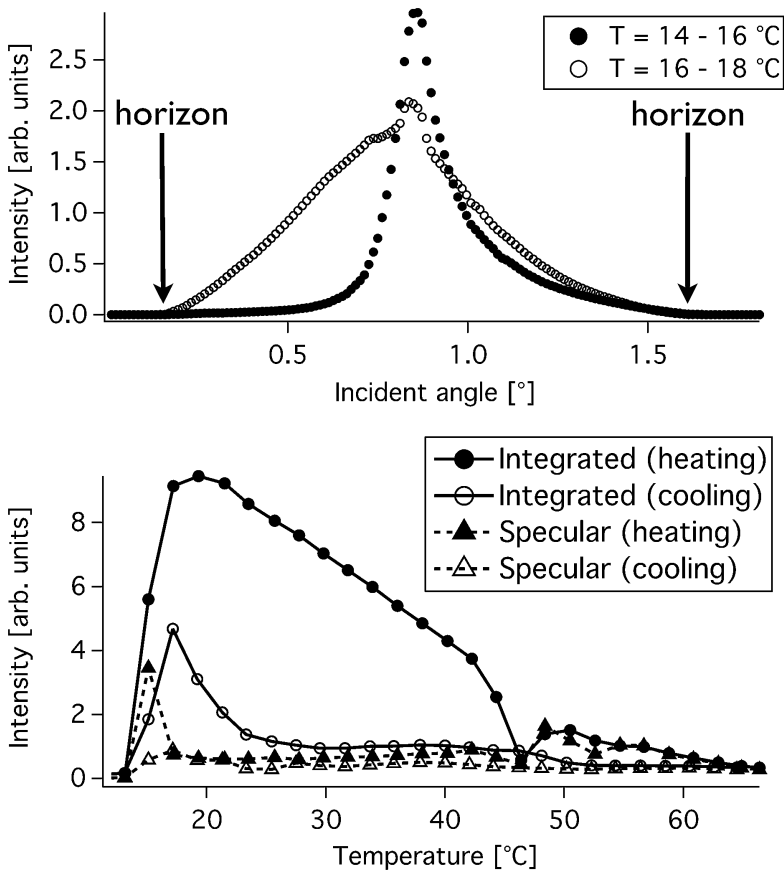

Fig. 5 (Upper panel) The Rocking intensity is plotted versus the incident beam angle for low temperatures in the cubic phase while heating the sample. The lower panel depicts the integrated intensity of each Rocking curve as well as the intensity of the specular part (lines are guide to the eyes)

smaller than the size of the symbols, can be as large as $100 \%$. The increased intensity at $15{ }^{\circ} \mathrm{C}$ relates to the fact that the diffuse and specular peak widths are similar and thus the heights of both peaks can not be separated. This indicates a large in-plane correlation length or crystallite size of more than $20 \mu \mathrm{m}$. In the present geometry intensity beyond the sample horizon, marked by the two arrows in the upper panel of Fig. 5, is not accessible. Converting the probed angular range to inplane momentum transfer $Q_{x}$ results in $6.8 \times 10^{-3} \mathrm{~nm}^{-1}$ or a length scale of $150 \mathrm{~nm}$. For smaller correlation lengths the peak will partly disappear from the measurement window. This implies that the intensity inside our window is directly related to the coherence of the micellar crystal. Following along this line Fig. 5, lower panel, depicts the integrated intensityt: plotted as circles versus temperature. In the cubic phases a distinct decrease of the intensity is visible indicating a decreasing correlation length for increasing temperature. A local minimum can be found at the cubic-hexagonal phase transition in the heating cycle at $46{ }^{\circ} \mathrm{C}$. Further, a reduced intensity mirrors a generally smaller correlation length $\S$ in the hexagonal phase compared to the cubic one as well as in the cooling cycle compared to the heating cycle.

Fig. 6 depicts the Rocking curves taken while heating and cooling for a temperature of $43{ }^{\circ} \mathrm{C}$. Apart from the increased

\$ The uncertainty in intensity, assuming the statistical error in the count rate, is smaller than the size of the symbols.

$\S$ Note: A higher correlation length would result in a reduced diffuse scattering as well but in that case higher intensity would be expected at the specular position.

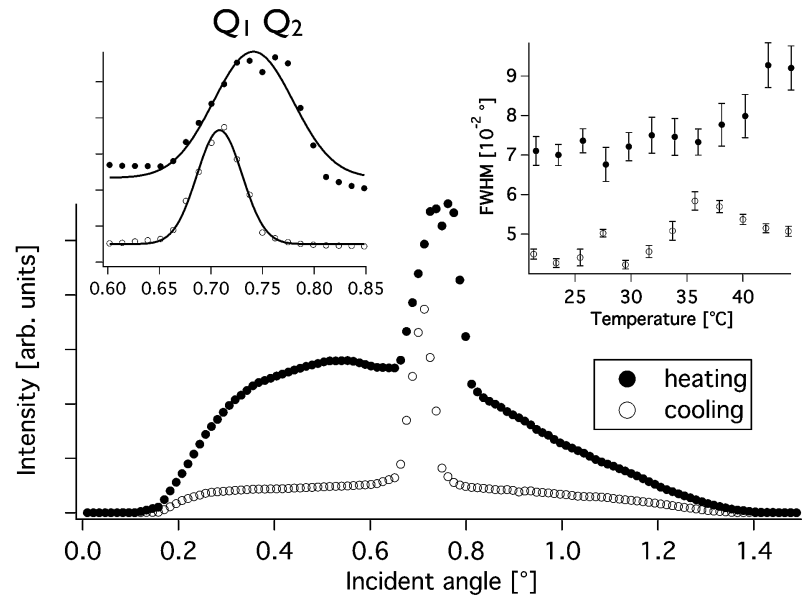

Fig. 6 Rocking intensity for a temperature of $43^{\circ} \mathrm{C}$ while heating and cooling the sample. Zoom into the narrow component of the Rocking curve (upper left panel). The lines represent a Gaussian fit to the data. The upper right panel depicts the FWHM extracted from a Gaussian fit to the narrow component.

diffuse scattering the sharp narrow specular component is broadened while heating. This is reflected in the FWHM extracted from a fit of one Gaussian function to the data and depicted in the upper right panel. The FWHM is systematically broader during heating. Additionally it turns out that the Gaussian fit has a larger error while heating the sample and the line shape fits worse as seen in the upper left panel representing a zoom into the specular region. The solid lines refer to the fit with one Gaussian line to the data. It can be seen that the fit is less reliable for the data taken during heating since the data actually show two peaks $\llbracket Q_{1}$ and $Q_{2}$. From the position of the two peaks $\|$ a difference in $d$-spacing along the surface normal of $0.8 \mathrm{~nm}$ can be calculated. Our model implies a coexistence of two phases with different lattice parameters, which are both stable rather than a continuous transition from one to another phase.

The colored (darker) symbols in Fig. 7 depict the $d$-spacing, extracted from fitting one Gaussian function with sloping background to the narrow component of the Rocking curves, plotted versus temperature for heating and subsequent cooling. A distinct temperature dependence is found. As a general trend the $d$-spacing increases with temperature. Nevertheless, we note several remarkable deviations. Between $17{ }^{\circ} \mathrm{C}$ and $25{ }^{\circ} \mathrm{C}$ the $d$ spacing of the structure remains almost constant and undergoes a distinct rise when further heating the sample. The $d$-spacing seems to have a local minimum at both cubic/

I Note: Both peaks are perfectly described by a Gaussian function assuming the theoretically calculated experimental resolution.

|| The position has been extracted from the maximum value in the data. The uncertainty in each measured point relating to the statistics in the count rate is smaller than $1 \%$ or the size of the symbols for all data points. An alternative fitting of the data with two Gaussian lines showed a strong correlation between the fitting parameters and did not give more reliable results. The uncertainty in the two $Q$ values is estimated from the increment in the incident beam angle, which is about one fifth of the beam divergence. 


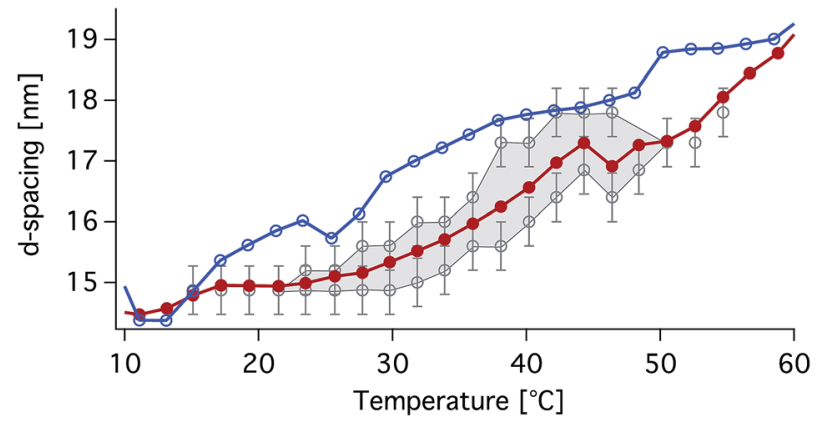

Fig. 7 Interlayer spacing along the $z$-direction extracted for different temperatures (lines are guide to the eyes). The blue and red symbols correspond to data taken for cooling and heating the sample, respectively.

hexagonal phase transition temperatures in the heating at $46^{\circ} \mathrm{C}$ and the cooling cycle at $25{ }^{\circ} \mathrm{C}$. However, since the intensity of the Bragg reflection is small during the reformation of the phases a final prove of this effect would need further more detailed measurements in the respective temperature ranges. During the cooling cycle the $d$-spacing is higher for the same temperatures. The error in the position of the fit for the cooling as well as for the heating cycle is below $1 \% * *$ of the value resulting in error bars smaller than the size of the symbols in Fig. 7. Alternatively to fitting one Gauss to the narrow component the two maxima visible in Fig. 6 can be determined for each temperature. The two $d$-spacings extracted in this way and as described above are represented by the light gray circles in the figure. Note, the jump in lattice parameter of $0.8 \mathrm{~nm}$ at the phase transition around $46{ }^{\circ} \mathrm{C}$ corresponds well to the difference between the two lattice parameters extracted from the double peak.

Our results imply, that the cubic phase is stable for temperatures between 14 and $25{ }^{\circ} \mathrm{C}$. For temperatures in the range from 25 to $46^{\circ} \mathrm{C}$ the cubic phase is still present but with a large number of stacking faults and hexagonal domains, which manifest in the broadening of the specular line and the increased diffuse scattering as seen in Fig. 6 . At $46^{\circ} \mathrm{C}$ the cubic phase turns fully into the hexagonal structure. The coexistence of hexagonal and cubic ordering in a temperature range above $25{ }^{\circ} \mathrm{C}$ under heating corresponds well to the phase behavior in the cooling cycle. Coming from high temperatures the sample enters the hexagonal phase at $48{ }^{\circ} \mathrm{C}$ and remains in that structure over a broad range of temperature. We note that in this hexagonal phase the width of the specular peak follows the limitations of the given resolution and no double peaks are visible. At $25{ }^{\circ} \mathrm{C}$ the sample undergoes a phase transition from hexagonal to cubic stacking. Our results indicate that the hexagonal ordering is the equilibrium structure above temperatures of $25{ }^{\circ} \mathrm{C}$ but during the heating cycle the sample gets trapped in the cubic phase since the micelles are sticky and can not rearrange.

** Errors are taken from the statistical error of the fitting routine and represent one sigma, $65 \%$ confidence.
The equilibrium structure of a thermodynamic system can be calculated by minimizing the free energy, $F=U-T S$, where $U, T$ and $S$ symbolize the internal energy (including the internal and configurational part), temperature and conformational entropy, respectively. For a system with two components the dimensionless parameter $\chi$ measuring the relative importance of the energy of interaction between the two components $A$ and $\mathrm{B}, \varepsilon_{\mathrm{AA}}, \varepsilon_{\mathrm{BB}}$ and $\varepsilon_{\mathrm{AB}}$ can be introduced:

$$
\chi=\frac{z}{2 k_{\mathrm{B}} T}\left(2 \varepsilon_{\mathrm{AB}}-\varepsilon_{\mathrm{AA}}-\varepsilon_{\mathrm{BB}}\right),
$$

with $z$ representing the number of nearest neighbors for each species A and B. By taking the interpretation of entropy in statistical mechanics, as a logarithmic measure of the density of states the entropy part in the free energy can be written as $S_{\text {mix }}$ :

$$
\frac{S_{\mathrm{mix}}}{k_{\mathrm{B}} T}=\phi_{\mathrm{A}} \ln \phi_{\mathrm{A}}+\phi_{\mathrm{B}} \ln \phi_{\mathrm{B}}+\chi \phi_{\mathrm{A}} \phi_{\mathrm{B}}
$$

where $\phi_{\mathrm{A}}$ and $\phi_{\mathrm{B}}$ denote the volume fraction of $\mathrm{A}$ and $\mathrm{B}$, respectively.

It has been reported that PEO as well as PPO undergo a conformational change when solved in water and with increasing temperature. ${ }^{24}$ As a result the parameter $\chi$ changes from values of 0.65 and 1.7 (in units of kT) for PEO and PPO, respectively, at lower temperatures to 5.6 and 8.5 at higher ones. ${ }^{29}$ However, the temperature at which this transition occurs is about $15{ }^{\circ} \mathrm{C}$ for PPO and $70{ }^{\circ} \mathrm{C}$ for PEO. Fig. 8 summarizes the expected phase separation calculated from eqn (2). With increasing temperature the system gets highly phase separating.

Svensson et al. calculated the phase diagram ${ }^{29}$ of $\mathrm{P} 123$ solved in water with a mean field lattice theory including the internal free energy, the configurational energy in the mixed system and the mixing conformational entropy. The theoretically predicted phase diagram is represented in Fig. 9, left panel. A hexagonal phase assuming rods is predicted but the cubic phase is reported to be unstable.

In ref. 29 the modified free energy was calculated for the construction of the phase diagram. Fig. 9, right panel, represents a plot extracted from ref. 29 representing the modified free energy for different polymer concentrations at a temperature of $29^{\circ} \mathrm{C}$. It turns out that in a wide concentration range the free energy of the cubic and the hexagonal phase is almost the

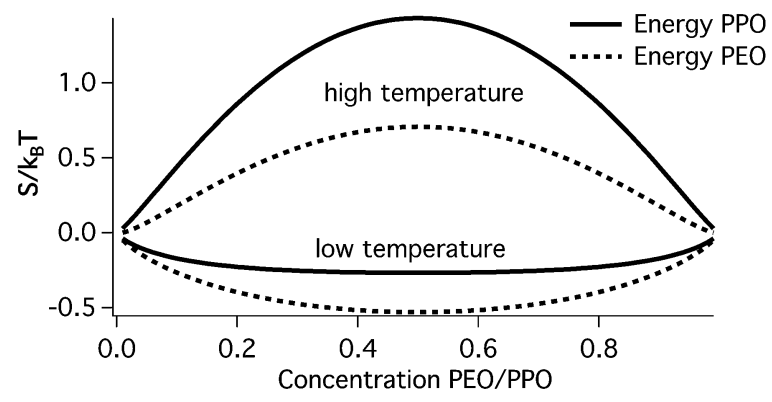

Fig. 8 Relative concentration of PEO and PPO solved in water in the low and high temperature configuration. 


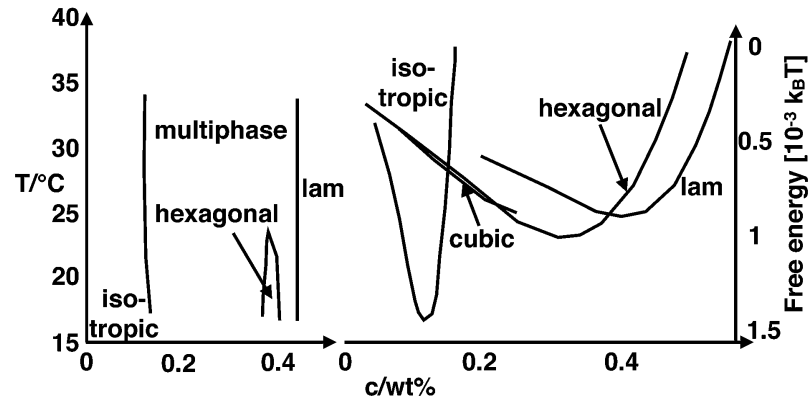

Fig. 9 Phase diagram of P123 solved in water calculated by mean-field lattice theory and reproduced from ref. 29.

same implying a coexistence of both phases. Moreover, both phases have the lowest free energy in the concentration range investigated in our study, even though Svensson et al. claim that the system should decompose into micelles and lamellas, assuming a linear superposition of these two phases. However, this is not reported experimentally (see Fig. 1).

In the theoretical study just mentioned the free energy was calculated for different structures by assuming a certain configuration and shape of the polymer micelles and the concentration of PEO, PPO and water inside the micelles was calculated. The polymer concentration in the core as well as in the shell increases for higher temperatures ${ }^{29}$ and with increasing phase separation the number of molecules per micelle increases. ${ }^{14}$ This implies an increasing size of the micelles and results in the larger $d$-spacing. For dilute systems the micelles additionally undergo changes in shape ${ }^{15}$ by accumulating free unimers and with an increasing number of unimers per micelle an ellipsoidal shape becomes more favorable.

The change in micellar shape was also reported for bulk samples. It is expected that at a surface ellipsoidal micelles orient with respect to the anisotropy of the solid boundary. For cubic dense packed spheres the relation between the nearest neighbor distance, $D_{\mathrm{MM}}$, and the distance between densely packed planes, $d_{\mathrm{fcc}(111)}$, is:

$$
d_{\mathrm{fcc}(111)}=D_{\mathrm{MM}} \frac{\sqrt{2}}{\sqrt{3}}
$$

For densely packed rods, with the long axis parallel to the surface plane, ${ }^{22}$ the distance between lattice planes along the surface normal is given by:

$$
d_{\text {hex }(100)}=D_{\text {MM }} \sin \left(60^{\circ}\right)=D_{\text {MM }} \frac{\sqrt{3}}{2}
$$

and the ratio between the two becomes:

$$
\frac{d_{\text {hex }(100)}}{d_{\mathrm{fcc}(111)}}=\frac{3}{2 \sqrt{2}} \approx 1.06
$$

for the same nearest neighbor distance. This implies that the $d$ spacing should increase by $6 \%$, when going from a dense packing of spheres to a dense packing of rods. For a $d$-spacing of

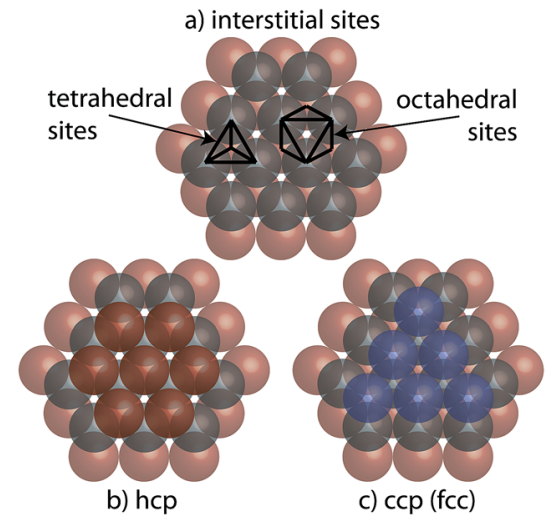

Fig. 10 Panel (a) shows the positions and surfaces of octahedral and tetrahedral sites in a close packed structure. Panels (b) and (c) depict the schematics of a hexagonal and cubic close packing sequence, respectively.

$15 \mathrm{~nm}$ this translates to a difference of $0.9 \mathrm{~nm}$ which is very close to the value extracted from the double peak.

Qualitatively the phase transition between cubic (panel c, Fig. 10) and hexagonal (panel b, Fig. 10) close packing can be illustrated when looking at the structural arrangements of the micelles. For close packed structures two interstitial sites, tetrahedral and octahedral, exist as depicted in Fig. 10 (panel a). For the following we assume polyhedra with plane surfaces. An octahedron has a volume, which is four times larger than the one of a tetrahedron but only twice the surface area. As a result, to cover the same volume and regarding the dense packing, octahedra have a two times smaller surface area. This implies that with increasing $\chi$ (demixing) the water will concentrate in the octahedral sites, since the surface to volume ratio is favorable at this position.

In the cubic structure the octahedral sites are linked via corners and edges but in the hexagonal structure they are additionally linked via planes. As a result the surface area of the octahedral sites is further reduced by $1 / 4$, since the octahedra are touching each other on every forth plane. This is seen as the white areas in Fig. 10 and as a result a hexagonal pattering of water rich rods can form. The total number of octahedral and tetrahedral sites in the cubic and hexagonal structure is the same. This results in a total reduction of the surface area of $1 / 8$ for the hexagonal close packing, since there are twice as many tetrahedral sites than octahedral ones. Since the calculated energy difference between the two phases is tiny this might be the driving force of the phase transition.

\section{Conclusion}

We performed a grazing incidence neutron scattering study of a micellar solution of the three block copolymer P123 solved in deuterated water. Rocking curves of the first order Bragg reflection were evaluated with respect to the position and width. We find different structural arrangements for continuously heating or cooling the sample. Initially, when heated from low temperatures spherical micelles form a cubic crystal. At 
intermediate temperatures the cubic phase coexists together with a hexagonal structure before fully turning into the hexagonal arrangement upon further heating. At the highest temperatures the crystalline structure melts. During cooling no coexistence is found but rather an abrupt transition from hexagonal to cubic at $25{ }^{\circ} \mathrm{C}$. We qualitatively relate our findings to changes in the micellar shape from spherical to elongated at higher temperatures together with a minimization of the polymer water interface. The results show the delicate correlation of temperature, micellar properties and type of structure and are consistent with previous studies but call for a revised theory including surface energy effects to explain the tiny differences in the free energy between cubic and hexagonal structures.

\section{Acknowledgements}

The authors thank Marco Walz for help during the preparation of the experiment as well as Andreas Magerl for intensive and interesting discussions. The authors gratefully acknowledge financial support from the BMBF (03ZA7BOC), the DFG grant MA801/13-1, as well as the Swedish research council under contract numbers (A0505501 and C0511501).

\section{References}

1 L. Qin, S. Park, L. Huang and C. A. Mirkin, Science, 2005, 309, 113.

2 S. Park, J.-H. Lim, S.-W. Chung and C. A. Mirkin, Science, 2004, 303, 348.

3 M. A. Hillmyer, Adv. Polym. Sci., 2005, 190, 137.

4 S. Förster, A. Timmann, C. Schellbach, A. Frömsdorf, A. Kronowski, H. Weller, S. V. Roth and P. Lindner, Nat. Mater., 2007, 6, 888.

5 R. A. L. Jones, Soft Condensed Matter, Oxford University Press, Oxford, 2002.

6 P. Lindner, Neutrons, X-rays and light: scattering methods applied to soft condensed matter, ed. P. Lindner, Elsevier, Amsterdam, 2002.

7 K. Mortensen, W. Brown and E. Jorgensen, Macromolecules, 1995, 28, 1458.
8 K. Mortensen, D. Schwahn and S. Janssen, Phys. Rev. Lett., 1993, 71, 1728.

9 K. Mortensen and Y. Talmin, Macromolecules, 1995, 28, 8829.

10 K. Mortensen, Macromolecules, 1997, 39, 503.

11 K. Mortensen, W. Brown and B. Norden, Phys. Rev. Lett., 1992, 68, 2336.

12 K. Mortensen and J. S. Pedersen, Macromolecules, 1993, 26, 805.

13 K. Mortensen, Curr. Opin. Colloid Interface Sci., 1998, 3, 12.

14 I. Goldmints, G.-E. Yu, C. Booth, K. A. Smith and T. A. Hatton, Langmuir, 1999, 15, 1651.

15 G. Wanka, H. Hoffmann and W. Ulbricht, Macromolecules, 1994, 27, 4145.

16 K. Mortensen, J. Phys.: Condens. Matter, 1996, 8, A103-A124.

17 P. Müller-Buschbaum, R. Cubitt and W. Petry, Langmuir, 2003, 19, 7778.

18 W. A. Hamilton, P. D. Butler, S. M. Baker, et al., Phys. Rev. Lett., 1994, 72, 2219.

19 M. Wolff, U. Scholz, R. Hock and A. Magerl, Phys. Rev. Lett., 2004, 92, 255501.

20 M. Wolff, A. Magerl and H. Zabel, Langmuir, 2009, 25(1), 64.

21 M. Wolff, A. Magerl and H. Zabel, J. Phys.: Condens Matter, 2005, 17, S3645.

22 M. Walz, M. Wolff, N. Voss, H. Zabel and A. Magerl, Langmuir, 2010, 26, 14391.

23 S. K. Sinha, E. B. Sirota, S. Garoff and H. B. Stanley, Phys. Rev. B: Condens. Matter Mater. Phys., 1994, 38, 2297.

24 G. Karlström, J. Phys. Chem., 1985, 89, 4962.

25 C. Guo, J. Wang, H.-z. Liu and J.-y. Chen, Langmuir, 1999, 15, 2703.

26 M. Wolff, K. Zhernenkov and H. Zabel, Thin Solid Films, 2007, 515, 5712.

27 M. Wolff, A. Magerl and H. Zabel, Eur. Phys. J. E: Soft Matter Biol. Phys., 2005, 16(2), 141.

28 D. van der Grinten, M. Wolff, H. Zabel and A. Magerl, Meas. Sci. Technol., 2008, 19, 034016.

29 M. Svensson, P. Alexandridis and P. Linse, Macromolecules, 1999, 32, 637. 